Editor's Note: These short reviews of a recent paper in the Journal, written exclusively by graduate students or postdoctoral fellows, are intended to summarize the important findings of the paper and provide additional insight and commentary. For more information on the format and purpose of the Journal Club, please see http://www.jneurosci.org/misc/ifa_features.shtml.

\title{
Protein Disulfide Isomerase and the Endoplasmic Reticulum in Amyotrophic Lateral Sclerosis
}

\author{
Adam K. Walker ${ }^{1,2}$ \\ ${ }^{1}$ Howard Florey Institute, Florey Neuroscience Institutes, and Centre for Neuroscience, The University of Melbourne, Parkville, Victoria 3010, Australia, \\ and ${ }^{2}$ Department of Biochemistry, La Trobe University, Bundoora, Victoria 3086, Australia \\ Review of Yang et al.
}

Amyotrophic lateral sclerosis (ALS) is a devastating neurodegenerative disease primarily affecting motor neurons. No previous family history exists in the majority of cases, but mutations in the gene encoding superoxide dismutase 1 (SOD1) cause $\sim 20 \%$ of familial disease. Although the mechanisms causing motor neuron degeneration in ALS remain unknown, recent findings highlight a critical role for the endoplasmic reticulum (ER) in disease pathogenesis. Protein misfolding in the ER lumen can activate signaling transduction pathways known collectively as the unfolded protein response (UPR), which occurs in mutant SOD1 models of ALS (Atkin et al., 2006), and in human sporadic disease (Atkin et al., 2008).

The UPR attempts to resolve ER stress by inhibiting protein translation, increasing production of chaperone proteins, and enhancing protein degradation; however, cell death is triggered if misfolded

Received Jan. 25, 2010; revised Feb. 4, 2010; accepted Feb. 5, 2010.

A.K.W. is supported by an Australian Postgraduate Award and an Australian Rotary Health scholarship. I thank Dr. Julie Atkin and Prof. Malcolm Horne for helpful comments on this manuscript. Related studies in the Atkin and Horne laboratories were supported in part by National Health and Medical Research Council of Australia Project Grants 454749 and 236805, Amyotrophic Lateral Sclerosis Association (United States), Motor Neurone Disease Research Institute of Australia, Bethlehem Griffiths Research Foundation, and a Henry H. Roth Charitable Foundation Grant for Motor Neurone Disease Research.

Correspondence should be addressed to Adam K. Walker, Florey Neuroscience Institutes, The University of Melbourne, Parkville, Victoria 3010, Australia. E-mail: adam.walker@florey.edu.au.

DOI:10.1523/JNEUROSCI.0408-10.2010

Copyright $\odot 2010$ the authors $\quad 0270-6474 / 10 / 303865-03 \$ 15.00 / 0$ proteins continue to accumulate. The UPR is activated in motor neurons of the most commonly used ALS model, highexpressing mutant SOD1 ${ }^{\mathrm{G} 93 \mathrm{~A}}$ transgenic mice, as early as postnatal day 5 , long before symptom onset at approximately day 90 (Saxena et al., 2009). Additionally, genetic ablation of the ER stress-activated transcription factor X-box-binding protein-1 significantly delays disease onset and increases survival in mutant SOD1 mice (Hetz et al., 2009). These results indicate that ER stress could be an early event in the pathogenic cascade in ALS.

One important UPR-induced chaperone is protein disulfide isomerase (PDI). PDI is an abundant enzyme that forms, breaks, and isomerizes disulfide bonds and is therefore an important cellular defense against protein misfolding. PDI is upregulated before symptom onset in spinal cords of mutant SOD $1{ }^{\text {G93A }}$ mice and rats and in human ALS postmortem tissue (Atkin et al., 2008). Although PDI can be protective against mutant SOD1 aggregation and toxicity, aberrant S-nitrosylation of critical active site cysteine residues likely inactivates the normal protective function of PDI in ALS spinal cords (Walker et al., 2010).

In a recent paper published in The Journal of Neuroscience, Yang et al. (2009) further investigated the role of PDI in ALS and identified reticulons, a family of integral ER membrane proteins of primarily ill-defined function, as important modulators of PDI subcellular localization in vitro. Furthermore, the authors showed that deletion of the gene encoding reticulon-4A,B (Rtn-4A,B, also known as NogoA,B) prevents PDI redistribution and accelerates degeneration and disease progression in SOD1 ${ }^{\mathrm{G} 93 \mathrm{~A}}$ mice. These findings suggest that reticulon expression and PDI localization could be important modulating factors in ALS.

Initially, the authors identified that Rtn-4A expression altered the distribution of proteins containing a lysine-aspartic acid-glutamic acid-leucine (KDEL) motif, which is present at the far $\mathrm{C}$ terminus of many ER-localized proteins to allow receptor-mediated ER retention. Rtn-4A expression in simian fibroblast COS-7 cells, which normally have no detectable Rtn-4A, redistributed KDEL proteins from a reticular pattern, reminiscent of the normal ER, to a more punctate pattern (Yang et al., 2009, their Fig. 1). Using antibodies directed against individual KDEL proteins, the authors showed that Rtn-4A expression changed PDI distribution to a high degree, whereas other KDEL proteins, such as Grp94 (glucose-regulated protein 94), calreticulin, and $\mathrm{BiP}$ (Ig binding protein), displayed little or no change in localization (Yang et al., 2009, their Fig. 2). Furthermore, expression of different isoforms of the four mammalian reticulon proteins, namely Rtn-1C, Rtn2A, Rtn-3, and Rtn-4C (NogoC), also caused PDI redistribution (Yang et al., 2009, their Fig. 3). Therefore, redistribution of PDI to ER subcompartments or 
other regions within the cell could be a previously unidentified function of the reticulon protein family.

How do reticulons mediate PDI redistribution? Little colocalization was observed between PDI and reticulon proteins, indicating that changes in PDI localization were not mediated by direct interaction (Yang et al., 2009, their Fig. 3). Reticulon involvement in microtubulebased intracellular and axonal transport processes implicated in many adult-onset neurodegenerative diseases, including ALS, could be one explanation (Morfini et al., 2009).

Interestingly, Rtn-3 is both anterogradely and retrogradely transported in axons, and overexpression of Rtn-3 causes an imbalance in axonal cargo transport (Shi et al., 2009). Whether other reticulons are also involved in microtubulebased transport remains to be seen, but mutation of the microtubule-associated protein mini spindles in Drosophila alters localization of both PDI and the Drosophila reticulon protein reticulon like-1 (Pokrywka et al., 2009). This suggests that reticulons affect microtubule-based axonal transport and that distribution of both PDI and reticulons depends on microtubule-based transport.

A second, although not mutually exclusive, explanation for the altered PDI distribution could be the effect of reticulons on ER morphology. Rtn-3 expression prevents the disruption of ER structure normally seen after nocodozole-induced microtubule depolymerization (Shibata et al., 2008). Furthermore, ER morphology defects have been proposed as a pathogenic effect of atlastin-1 mutations, which cause a subset of hereditary spastic paraplegia, another disease affecting motor neurons (Hu et al., 2009). In addition to interacting with Rtn-4A, atlastin-1 interacts with the microtubule-severing protein spastin, and spastin mutations cause another subset of hereditary spastic paraplegia (Evans et al., 2006). Overall, these findings indicate that microtubulebased transport and ER structural changes are linked to both reticulons and other proteins involved in diseases affecting motor neurons. Indeed, the ALS-linked mutant VAPB (vesicle-associated membrane protein-associated protein B), a microtubule-associated protein, induces formation of inclusions characterized as a form of restructured organized smooth ER, containing a subset of ER-derived proteins (Fasana et al., 2010). A pathway involving changes to ER structure and protein distribution, mediated by reticu- lons and microtubules, could be important for motor neuron survival.

Yang et al. (2009) further confirmed that reticulons cause PDI redistribution in mouse motor neurons in vivo. Genetic deletion of Rtn-4A,B resulted in a more homogenous distribution of PDI with fewer PDI puncta in spinal motor neurons compared with those of wild-type mice, without any change in distribution of the ER transmembrane protein calnexin (Yang et al., 2009, their Fig. 5). Conversely, Rtn-4A overexpression in motor neurons increased PDI puncta formation, again without change in calnexin distribution (Yang et al., 2009, their Fig. 5).

To demonstrate that reticulon expression affected ALS disease onset and progression, the authors showed that genetic deletion of Rtn-4A,B accelerated axonal degeneration in spinal cords of transgenic SOD $1^{\text {G93A }}$-expressing mice (Yang et al., 2009, their Fig. 6). Additionally, SOD1 G93A mice with deletion of one or both Rtn- 4 A,B alleles reached disease end-stage significantly earlier than controls, with a decrease in survival of up to $13 \mathrm{~d}$ in $\operatorname{Rtn}-4 A, B^{+/-}$ mice and $20 \mathrm{~d}$ in $\operatorname{Rtn}-4 A, B^{-/-}$mice (Yang et al., 2009, their Fig. 8). Motor performance was also decreased and weight loss was accelerated in SOD ${ }^{\text {G93A }}$ mice with Rtn-4A,B deletion. These results suggest that Rtn$4 \mathrm{~A}, \mathrm{~B}$ protects against SOD ${ }^{\mathrm{G} 93 \mathrm{~A}}$-mediated neuronal degeneration, although future demonstration of a beneficial effect by reticulon overexpression in SOD ${ }^{\text {G93A }}$ mice is important to confirm this finding.

Whether reticulon-mediated PDI redistribution was the sole factor modulating disease course in SOD $1^{\text {G93A }}$ mice with Rtn-4A,B ablation remains unclear. Another possibility is that reticulon deletion affected ER stress signaling, which indirectly affected animal survival. The authors reported that the UPR was not altered with overexpression of Rtn-1C, Rtn-2A, Rtn-3, Rtn-4A, or Rtn-4C in COS-7 cells (Yang et al., 2009, their Fig. 4). However, UPR induction was seen previously in neuroblastoma cells but not in COS-7 cells, with mutant SOD1 expression (Atkin et al., 2006). Therefore, it would be pertinent to investigate the effect of reticulons on the UPR in neuronal cell culture and in the reticulon overexpressing and deletion mouse lines used in this study. Importantly, previous studies showed that Rtn-1C overexpression increased susceptibility to ER stress in neuroblastoma cells (Di Sano et al., 2007), whereas ER stress-induced Rtn-3 expression can be protective against various insults (Wan et al., 2007). The role of reticulons in ER stress signaling, whether neutral, protective, or detrimental, thus remains uncertain (Teng and Tang, 2008).

Another unanswered question is whether reticulon-mediated redistribution is specific for PDI or involves other ER proteins not investigated in this study. The authors note that expression of both Rtn-1C and Rtn-4C caused redistribution of the PDI family member ERp57, albeit to a lesser extent than PDI itself (Yang et al., 2009, their supplemental Fig. 1, available at www.jneurosci.org as supplemental material). Upregulation of ERp57 was found previously in both SOD $1^{\mathrm{G} 93 \mathrm{~A}}$ mice and rats, indicating that other PDI family members are involved in disease (Atkin et al., 2006). It is therefore important to identify which other ER proteins show altered distribution in the reticulon deletion and overexpression mice.

Finally, an issue not directly assessed in this study is the functional consequences of PDI redistribution. PDI is highly expressed in spinal cord motor neurons (Atkin et al., 2006) and undergoes aberrant posttranslational modification in ALS (Walker et al., 2010), indicating that PDI function could affect motor neuron survival. Reticulon-mediated PDI redistribution could lead to an increase in PDI activity as a result of altered protein interactions or increased availability of substrates. SOD1 could be one of the substrates affected by PDI redistribution, and it would be interesting to investigate whether mutant SOD1 aggregation was exacerbated by Rtn-4A,B deletion in the SOD $1{ }^{\text {G93A }}$ mice. It is possible that the interaction of PDI with other substrate proteins not yet identified could also be involved in disease.

In conclusion, Yang et al. (2009) provide strong evidence for a role of reticulon proteins in modulating mutant SOD1linked ALS, possibly by altering PDI subcellular distribution. Additional work is needed to define the microtubule involvement in changes to ER structure, which could link reticulon function with transport failures in ALS. Whether ER stress signaling, which is an early event in ALS, is involved in reticulon-mediated motor neuron protection also remains unclear. Nonetheless, this study identifies reticulons and reticulon-mediated ER changes, including PDI redistribution, as potential therapeutic targets in ALS.

\section{References}

Atkin JD, Farg MA, Turner BJ, Tomas D, Lysaght JA, Nunan J, Rembach A, Nagley P, Beart PM, Cheema SS, Horne MK (2006) Induction of 
the unfolded protein response in familial amyotrophic lateral sclerosis and association of protein-disulfide isomerase with superoxide dismutase 1. J Biol Chem 281:30152-30165.

Atkin JD, Farg MA, Walker AK, McLean C, Tomas D, Horne MK (2008) Endoplasmic reticulum stress and induction of the unfolded protein response in human sporadic amyotrophic lateral sclerosis. Neurobiol Dis 30:400-407.

Di Sano F, Fazi B, Tufi R, Nardacci R, Piacentini M (2007) Reticulon-1C acts as a molecular switch between endoplasmic reticulum stress and genotoxic cell death pathway in human neuroblastoma cells. J Neurochem 102:345353.

Evans K, Keller C, Pavur K, Glasgow K, Conn B, Lauring B (2006) Interaction of two hereditary spastic paraplegia gene products, spastin and atlastin, suggests a common pathway for axonal maintenance. Proc Natl Acad Sci U S A 103:10666-10671.

Fasana E, Fossati M, Ruggiano A, Brambillasca S, Hoogenraad CC, Navone F, Francolini M, Borgese N (2010) A VAPB mutant linked to amyotrophic lateral sclerosis generates a novel form of organized smooth endoplasmic reticulum. FASEB J. Advance online publication.
Retrieved Jan. 25, 2010. doi:10.1096/fj. 09-147850.

Hetz C, Thielen P, Matus S, Nassif M, Court F, Kiffin R, Martinez G, Cuervo AM, Brown RH, Glimcher LH (2009) XBP-1 deficiency in the nervous system protects against amyotrophic lateral sclerosis by increasing autophagy. Genes Dev 23:2294-2306.

Hu J, Shibata Y, Zhu PP, Voss C, Rismanchi N, Prinz WA, Rapoport TA, Blackstone C (2009) A class of dynamin-like GTPases involved in the generation of the tubular ER network. Cell 138:549-561.

Morfini GA, Burns M, Binder LI, Kanaan NM, LaPointe N, Bosco DA, Brown RH Jr, Brown H, Tiwari A, Hayward L, Edgar J, Nave KA, Garberrn J, Atagi Y, Song Y, Pigino G, Brady ST (2009) Axonal transport defects in neurodegenerative diseases. J Neurosci 29:1277612786.

Pokrywka NJ, Payne-Tobin A, Raley-Susman KM, Swartzman S (2009) Microtubules, the ER and Exu: new associations revealed by analysis of mini spindles mutations. Mech Dev 126:289-300.

Saxena S, Cabuy E, Caroni P (2009) A role for motoneuron subtype-selective ER stress in disease manifestations of FALS mice. Nat Neurosci 12:627-636.
Shi Q, Hu X, Prior M, Yan R (2009) The occurrence of aging-dependent reticulon 3 immunoreactive dystrophic neurites decreases cognitive function. J Neurosci 29:5108-5115.

Shibata Y, Voss C, Rist JM, Hu J, Rapoport TA, Prinz WA, Voeltz GK (2008) The reticulon and DP1/Yoplp proteins form immobile oligomers in the tubular endoplasmic reticulum. J Biol Chem 283:18892-18904.

Teng FY, Tang BL (2008) Cell autonomous function of Nogo and reticulons: the emerging story at the endoplasmic reticulum. J Cell Physiol 216:303-308.

Walker AK, Farg MA, Bye CR, McLean CA, Horne MK, Atkin JD (2010) Protein disulphide isomerase protects against protein aggregation and is S-nitrosylated in amyotrophic lateral sclerosis. Brain 133:105-116.

Wan Q, Kuang E, Dong W, Zhou S, Xu H, Qi Y, Liu Y (2007) Reticulon 3 mediates Bcl-2 accumulation in mitochondria in response to endoplasmic reticulum stress. Apoptosis 12: 319-328.

Yang YS, Harel NY, Strittmatter SM (2009) Reticulon-4A (Nogo-A) redistributes protein disulfide isomerase to protect mice from SOD1-dependent amyotrophic lateral sclerosis. J Neurosci 29:13850-13859. 